\title{
Converged Wired and Wireless Services in Next Generation Optical Access Networks
}

\author{
Colm Browning ${ }^{1}$, Arman Farhang ${ }^{1}$, Arsalan Saljoghei ${ }^{2}$, Nicola Marchetti ${ }^{2}$, Vidak Vujicic ${ }^{1}$, \\ Linda E. Doyle ${ }^{2}$, and Liam P. Barry ${ }^{1}$ \\ ${ }^{I}$ Department of Electronic Engineering, Dublin City University, Glasnevin, Dublin 9, Ireland \\ ${ }^{2}$ CONNECT Research Centre, Dunlop Oriel House, Trinity College Dublin, Dublin 2, Ireland \\ Tel:+3531700 7747, email: colm.browning@dcu.ie
}

\begin{abstract}
$5 \mathrm{G}$ communications will require the effective transmission of new radio signals through fiber networks, in order to facilitate the proliferation of antenna sites as well as greater pooling of resources. Next generation optical access networks can provide an efficient platform for mobile $\mathrm{x}$-haul. Techniques discussed in this paper shows how both wired and 5G wireless services may be converged over a single fiber infrastructure, and how optical networking can be harnessed in order to provide flexible millimeter-wave radio-over-fiber.
\end{abstract}

Keywords: optical access networks, $5 \mathrm{G}$, PON convergence, radio-over-fiber.

\section{INTRODUCTION}

With $5^{\text {th }}$ generation $(5 \mathrm{G})$ mobile communications on the horizon - entailing greater signal bandwidths and a greatly increased number of connected devices [1] - there has been much discussion recently as to what, from a networking perspective, will be pertinent. It seems likely that an ultra-dense deployment of small cell antenna sites, coupled with increased pooling of resources through cloud radio access networking (C-RAN), will be employed in order provide the required speeds in an efficient manner [1,2].

In today's mobile base stations, transmission of mobile data over optical fiber takes place between the baseband unit (BBU) and the remote radio head (RRH) - known as fronthaul - and is governed by industry agreements (CPRI, OBSAI). A move toward C-RAN architectures will see the pooling of the digital operations carried out by the BBU at a central location, allowing for the efficient distribution of RRHs. Due to C-RAN topology, and the requirement for increased fronthaul distances, it follows that passive optical networks (PON) have been proposed as an efficient means for $5 \mathrm{G}$ transport as they offer pre-existing infrastructure, high capacity, low latency and flexibility through the use of wavelength division multiplexing (WDM) and time division multiplexing (TDM) $11,3,4]$. Figure 1 shows a converged WDM based PON. BBUs are consolidated at a central office with optical line terminals (OLT) and downstream transmission of wired and wireless services can be provisioned through WDM networking or can be transmitted in tandem on a single wavelength to co-located optical networking units (ONU) and RRHs.

The following sections will discuss some optical networking options which will facilitate the development of effective $5 \mathrm{G}$ communications, in a flexible manner, over optical access.

\section{5G OVER FIBER NETWORK OPTIONS}

\subsection{Waveforms}

Considerable attention has been paid to improving the characteristics of the $4 \mathrm{G}$ long term evolution (LTE) standard waveform orthogonal frequency division multiplexing (OFDM) - particularly with regard to its strict subcarrier orthogonality requirements which make it susceptible to timing synchronization and frequency offset errors [5].

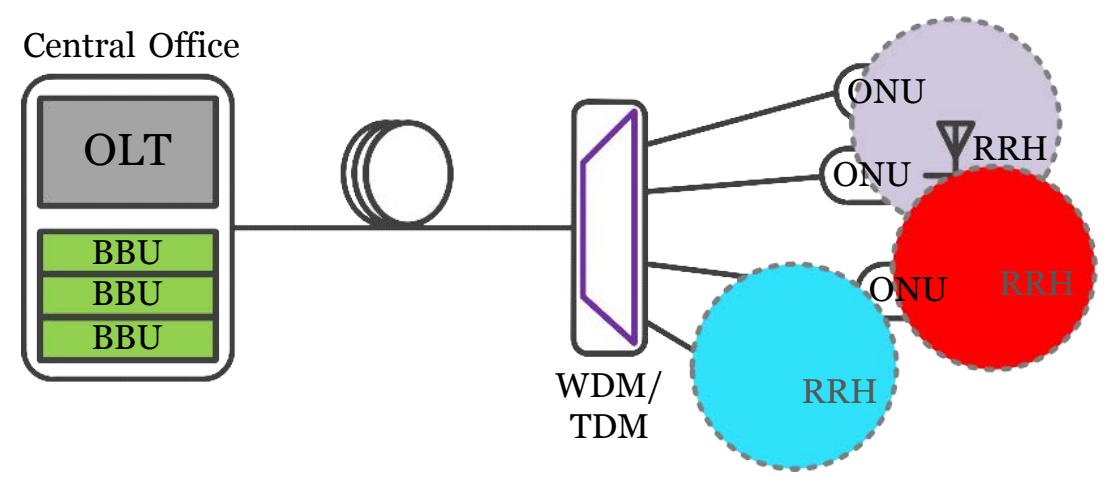

Figure 1. Network topology of a converged wired access with $5 G$ mobile fronthaul in a WDM/TDM PON. 


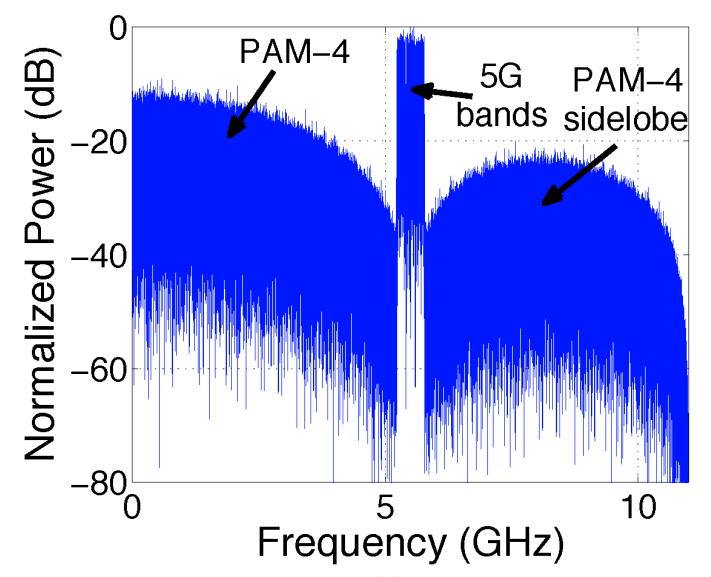

(a)

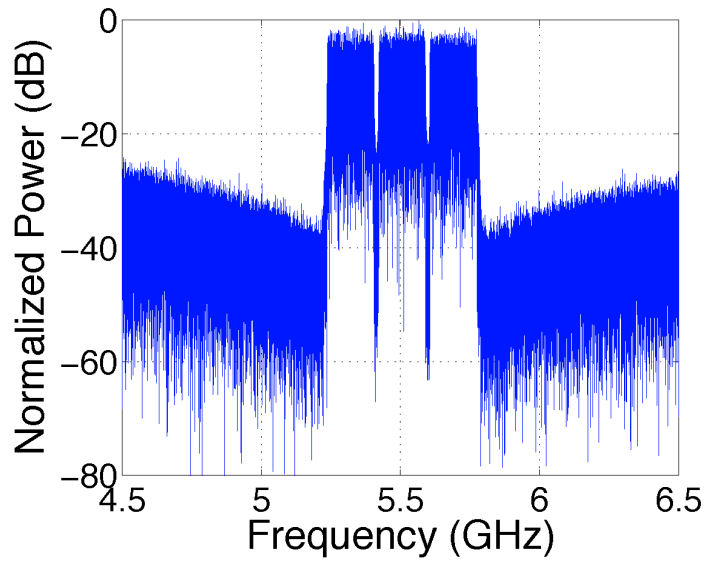

(b)

Figure 2: (a) Spectrum of three UF-OFDM bands with a 5.5GBaud PAM-4 and (b) a zoomed version.

5G candidate waveforms include universally filtered OFDM (UF-OFDM), generalized frequency division multiplexing (GFDM) and filter-bank multi-carrier (FBMC). These waveforms are similar to OFDM in their digital implementation but they employ various filtering and phase offset measures in an effort to lessen the orthogonality requirement and increase robustness. An excellent description of these waveforms can be found in [5] and [6]. Studies of these waveforms must be extended to the optical domain in order to assess their suitability for fiber transmission and ease of convergence with other services.

\subsection{Converged Services RoF}

In a next generation access scenario where an ONU and RRH are co-located, it may be advantageous to transmit combined wired and wireless services on a single wavelength channel. Work presented in [7] shows how by upgrading 10G fixed line services to four level pulse amplitude modulation (PAM-4), a spectral null can be created into which wireless channels can be inserted at an intermediate frequency (IF-RoF). UF-OFDM's low out-ofband emission is exploited to achieve effective convergence, spectral representations of which can be seen in Figs. 2(a) and 2(b) taken from [7].

\subsection{Millimeter-Wave RoF}

Due to the availability of spectrum, and the potential proliferation of 'small cell' antenna sites, millimeter-wave (mm-wave) frequencies have attracted considerable attention for 5G communications [8]. Frequency bands around 28, 60, and $90 \mathrm{GHz}$ may offer the required spectrum for multi gigabit communications, but the fact remains that optical fronthaul and/or backhaul must still be performed in an efficient manner. One method used to generate mm- wave RoF is known as optical heterodyning [9], a conceptual representation of which is presented in fig. 3. Rather than converge the wireless service with a wired service, as described above, the wireless data is modulated onto an optical carrier, and an additional optical carrier is added to the spectrum; offset by the desired mm-wave frequency. Phase correlation is required between these carriers and methods employing low linewidth external cavity lasers (ECL) [10], dual wavelength sources [8] and optical frequency combs [11] have been proposed as optical sources for these systems. The spectral components are transmitted through the optical access network and beating at the receiver photo-diode results in mm-wave frequency wireless data which can then be radiated. The use of tuneable optical components allows this heterodyne technique to offer flexible mm-wave RoF networking as frequencies in any band may be accessed, provided sufficient electrical bandwidth is available at the receiver.

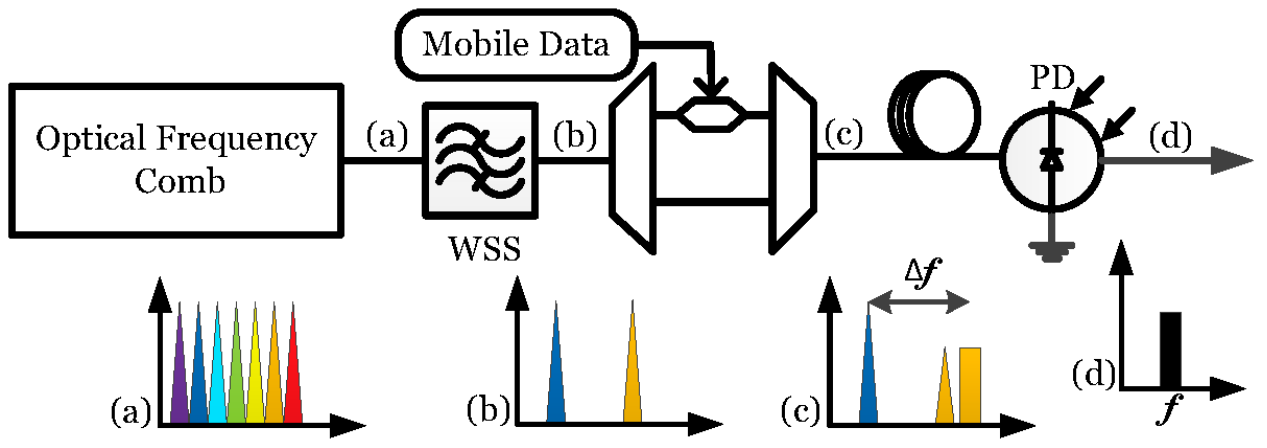

Figure 3. Concept of optical heterodyne mm-wave generation using an optical frequency comb. 


\section{CONCLUSION}

Requirements for high bandwidth, network flexibility and increased resource centralization associated with C-RAN based $5 \mathrm{G}$ mobile networks, entails a paradigm shift with regard to the optical portion of radio access. The convergence of wired and wireless services in future optical access networks will provide an efficient means of meeting the mobile transport requirements for antenna deployment and high capacity.

Additionally, techniques such as heterodyning, in combination with tunability in the optical domain, can provide flexible mm-wave RoF solutions which facilitate the necessary exploitation of available radio spectrum - a key requirement for $5 \mathrm{G}$ communications.

\section{ACKNOWLEDGEMENTS}

This publication has emanated from research supported in part by a research grant from Science Foundation Ireland (SFI) and is co-funded under the European Regional Development Fund under Grant Number 13/RC/2077. The work has also been jointly supported through the SFI US-Ireland project (15/US-C2C/I3132) and IPIC (12/RC/2276) research grants.

\section{REFERENCES}

[1] J. G. Andrews, S. Buzzi, W. Choi, S. V. Hanly, A. Lozano, A. C. K. Soong, and J. C. Zhang, "What will 5G be?," IEEE Journal on Selected Areas in Communications, vol. 32, pp. 1065-1082, Jun. 2014.

[2] R. G. Stephen and R. Zhang, "Joint millimeter-wave fronthaul and OFDMA resource allocation in ultradense CRAN," IEEE Transactions on Communications, vol. 65, pp. 1411-1423, Mar. 2017.

[3] S. Xu, S. Xu, and Y. Tanaka, "Dynamic resource reallocation for 5G with OFDMA in multiple user MIMO RoF- WDM-PON," in Proc. 2015 21st Asia-Pacific Conference on Communications (APCC), pp. 480-484, Oct. 2015.

[4] X. Yu, J. B. Jensen, D. Zibar, C. Peucheret, and I. T. Monroy, "Converged wireless and wireline access system based on optical phase modulation for both radio-over-fiber and baseband signals," IEEE Photonics Technology Letters, vol. 20, pp. 1814-1816, Nov. 2008.

[5] B. Farhang-Boroujeny and H. Moradi, "OFDM inspired waveforms for 5G," IEEE Communications Surveys Tutorials, vol. 18, pp. 2474-2492, Fourth quarter 2016.

[6] V. Vakilian, T. Wild, F. Schaich, S. ten Brink, and J. F. Frigon, "Universal-filtered multi-carrier technique for wireless systems beyond LTE," in Proc. 2013 IEEE Globecom Workshops, pp. 223-228, Dec. 2013.

[7] C. Browning, A. Farhang, A. Saljoghei, N. Marchetti, V. Vujicic, L. Doyle, and L. Barry, "5G wireless and wired convergence in a passive optical network using UF-OFDM and GFDM," arXiv:1703.01956, Mar. 2017.

[8] S. Alavi, M. Soltanian, I. Amiri, M. Khalily, A. Supa'at, and H. Ahmad, "Towards 5G: A photonic based millimeter wave signal generation for applying in 5G access fronthaul," Scientific Reports, vol. 6, p. 19891, 2016.

[9] T. Shao, M. Beltrán, R. Zhou, P. M. Anandarajah, R. Llorente, and L. P. Barry, “60 GHz radio over fiber system based on gain-switched laser," J. Lightwave Technol., vol. 32, pp. 3695-3703, Oct. 2014.

[10] S. Rommel, R. Puerta, J. J. V. Olmos, and I. T. Monroy, "Capacity enhancement for hybrid fiber-wireless channels with $46.8 \mathrm{Gbit} / \mathrm{s}$ wireless multi-cap transmission over $50 \mathrm{~m}$ at W-band," in Proc. Optical Fiber Communication Conference, p. M3E.5, Optical Society of America, 2017.

[11] E. P. Martin, T. Shao, V. Vujicic, P. M. Anandarajah, C. Browning, R. Llorente, and L. P. Barry, "25-Gb/s OFDM 60-GHz radio over fiber system based on a gain switched laser," Journal of Lightwave Technology, vol. 33, pp. 1635-1643, Apr. 2015. 